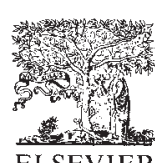

ELSEVIER

\title{
Genetic Polymorphisms in CYP3A5 and MDR1 Genes and Their Correlations With Plasma Levels of Tacrolimus and Cyclosporine in Renal Transplant Recipients
}

\author{
J. Mendes, A. Martinho, O. Simoes, A. Mota, L. Breitenfeld, and L. Pais
}

\begin{abstract}
Immunosuppressive drugs, such as tacrolimus (FK506) and cyclosporine (CsA), play an essential role in graft survival, preventing rejection. Large interindividual differences in drug-metabolizing enzymes as well as in drug transporters make the task of reaching the optimal concentrations difficult. The bioavailability of CsA and FK506 seems to be associated with the cytocrhome P450 IIIA (CYP3A) gene. It has also been described that the Multi Drug Resistance 1 (MDR1) gene that encodes for polyglycoprotein-P (P-gp) may influence the metabolizing action of FK506 and CsA. Therefore, we sought, to correlate single nucleotide polymorphisms (SNPs) in the CYP3A and MDR1 genes with the concentrations of FK506 and CsA. For this purpose we analyzed 2 groups of renal transplant recipients by sequencing: one receiving a CsA immunosuppressive regime, and other, an FK506-immunosuppression. This study showed that subjects in the FK506 group who had encoded the $1236 \mathrm{C}>\mathrm{T}$ substitution in the MDR1 gene displayed $44.4 \%$ higher drug concentrations compared with ("wild-type") individuals. Individuals carrying the 2677G $>$ T,A mutation showed FK506 concentrations that were $44.7 \%$ higher than the wild-type individuals. Concerning the CsA group, individuals carrying the $22915 \mathrm{~A}>\mathrm{C}$ substitution displayed CsA concentrations $52.1 \%$ higher than wild-type individuals.
\end{abstract}

$\mathrm{P}$ HARMACOGENETICS applies information related to genetic variability in relation to pharmacodynamics and metabolism of drugs. Some variations, referred to as single nucleotide polymorphisms (SNPs), when present in drug-metabolizing enzymes or drug transporters, may significantly alter the dosing of a given drug. Cytochrome $\mathrm{P} 450$ IIIA $(C Y P 3 A)$, and in particular its variant $C Y P 3 A 5$, plays a key role in metabolism of immunosuppressive drugs, such has tacrolimus (FK506) and cyclosporine (CsA), as well as a great variety of other substrates. ${ }^{1}$ Also, the drug transporter encoded by the Multi Drug Resistance (MDR1) gene, P-glycoprotein, which is responsible for actively exporting substrates from the inside of the cell to the exterior environment, ${ }^{2}$ plays a role. Several authors have described FK506 and CsA to be substrates for P-glycoprotein, making the genetic characterization of this gene of great relevance. ${ }^{1}$ In this study, we have focused on the third exon of the CYP3A5 gene. Polymorphisms in this region have been reported to have a relation to altered FK506 requirements., ${ }^{3,4}$ As regards the MDR1 gene, we analyzed a $2677 \mathrm{G}>\mathrm{T}$,A substitution in exon 21 and a $1236 \mathrm{C}>\mathrm{T}$ substitution in exon 12, both of which other studies have shown to significantly contribute to altered concentrations of immunosuppressants. $^{5}$

\section{MATERIALS AND METHODS}

Peripheral blood samples were collected from 2 groups of patients who underwent cadaveric renal transplantation. One group of 30 individuals (13 females and 17 males) received CsA-based immunosuppression regime; another 30 individuals (18 females and 12 males), FK506.

DNA was extracted using MagAttract DNA Blood Midi M48 Kit (Qiagen, Valencia, Calif). Amplification and sequencing primers

From the Centro de Histocompatibilidade do Centro (J.M., A.M., O.S., L.P.); the Unidade de Transplantação Renal dos Hospitais da Universidade de Coimbra (A.M.); and the Centro de Investigação das Ciências da Saúde da Universidade da Beira Interior (L.B.), Coimbra, Portugal.

Address reprint requests to Joao Fernando Pereira Mendes, Casal das Fontainhas Cruz dos Morouços, 3040-113 Coimbra, Portugal. E-mail: joaomendes77@hotmail.com

(c) 2009 by Elsevier Inc. All rights reserved. 360 Park Avenue South, New York, NY 10010-1710 


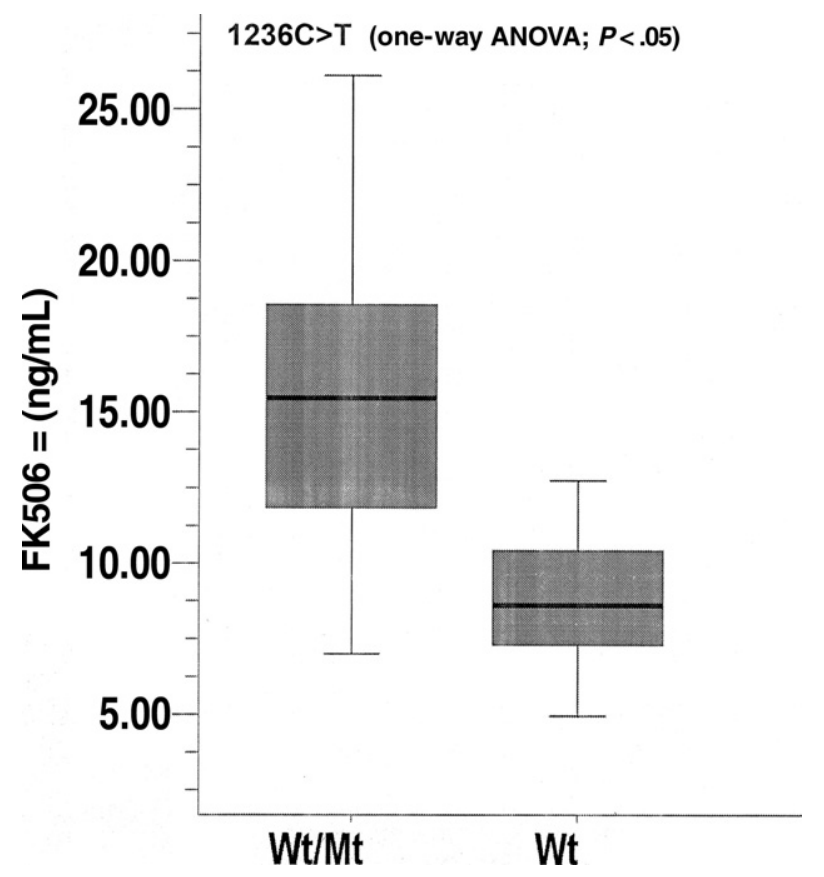

Fig 1. Correlation between levels of FK506 $(\mathrm{ng} / \mathrm{mL})$ and the SNP $1236 C>T$ in exon 12 of the MDR1 gene. Concentration distribution in accordance with the allelic variation. Wt, wild-type genotype; Mt, mutant genotype.

were obtained from Invitrogen. For genotype determination of exon 3 in the CYP3A5 gene, a forward $3^{\prime}$-CTTAACGAATGCTCTACTGTC-5' primer was used, along with the reverse $3^{\prime}$-ACACCCAGGAAGCCAGAC-5' primer. Regarding the MDR1 gene, genotyping of exon 12 was achieved with a forward primer $3^{\prime}$ TCCTGTGTCTGTGAATTGCCTTG-5' and reverse $3^{\prime}$-GCTGATCACCGCAGTCTAGCTCGC-5' ${ }^{\prime}$. Exon 21 of the MDR1 gene was genotyped with the forward $3^{\prime}$-GCAGGCTATAGGTTCCAGGCT-5' primer and with the reverse $3^{\prime}$-AACAGCCGGGTGGTGTCA-5' primer. Amplification polymerase chain reaction (PCR) was performed in a final volume of $50 \mu \mathrm{L}$ using $60 \mathrm{ng}$ of genomic DNA with $10 \mathrm{pmol}$ of each primer and $1.75 \mathrm{U}$ of Taq polymerase (Promega, Madison, Wisc). PCR products were sequenced with BigDye Terminator V.1.1. (Applied Biosystems, Foster City, Calif) in an ABI PRISM Genetic Analyzer (Applied Biosystems). The SNP analysis resultant from sequencing was correlated with the concentrations of FK506 and CsA using a one-way ANOVA test. Considering that the initial dose was given as $0.15 \mathrm{mg} / \mathrm{kg}$ for FK506 and $8 \mathrm{mg} / \mathrm{kg}$ for CsA, the first measurement of $\mathrm{C}_{0}(\mathrm{ng} / \mathrm{mL})$, after intravenous administration, was taken in account.

\section{RESULTS}

After SNP genotype analysis, we observed that the CsA group showed a $50 \%$ frequency of the SNP 22915A $>$ C in exon 3 of the $C Y P 3 A 5$ gene $(n=26)$. In the MDR1 gene, the SNP $1236 \mathrm{C}>\mathrm{T}$ occurred with a $40.74 \%$ frequency rate $(n=27)$ and for the SNP $2677 \mathrm{G}>\mathrm{T}, \mathrm{A}$, the frequency was $31.82 \%(n=22)$. For the FK506 group, the SNP in the MDR1 gene $1236 \mathrm{C}>\mathrm{T}$ occurred at a $34.78 \%(n=23)$ rate, and the $2677 \mathrm{G}>$ T,A substitution, $38.89 \%(n=18)$. The correlation of these data with concentrations of FK506 and CsA revealed that those receiving FK506 and were heterozygous for the SNP 1236C $>$ T showed concentrations $44.4 \%$ higher $(P<.05)$ than those of wild-type individuals (Fig 1). For the same group of individuals, those heterozygous for the 2677T $>$ A substitution, showed FK506 44.7\% higher $(P<.05)$ values than wild-type individuals (Fig 2$)$. For the CsA group, we observed that the 22915A $>C$ substitution contributed a $52.1 \%$ higher concentration of CsA than wild-type individuals (Fig 3).

\section{DISCUSSION}

The characterization of genes with a relation to the pharmacodynamics and metabolism of drugs displaying a narrow therapeutic index, such as immunosuppressants, is of great use to clinicians. In this manner, and with the growing knowledge of a vast number of SNPs associated with metabolizing enzymes, as well as with drug transporters, it may be possible to determine an individual's genetic profile for a metabolizing capacity related to a specific drug. In this study, we demonstrated that individuals heterozygous for the $1236 \mathrm{C}>\mathrm{T}$ and $2677 \mathrm{G}>\mathrm{T}$,A SNPs in the MDR1 gene show less efficient metabolism concerning FK506 compared with those displaying a wild-type genotype. Thus ideally they need lesser amounts of a given drug to reach the same concentrations. The same consideration is possible to establish for individuals heterozygous for the 22915A $>$ C SNP in the CYP3A5 gene, in relation to the use of CsA. The pharmacogenetic approach to metabolism represents an

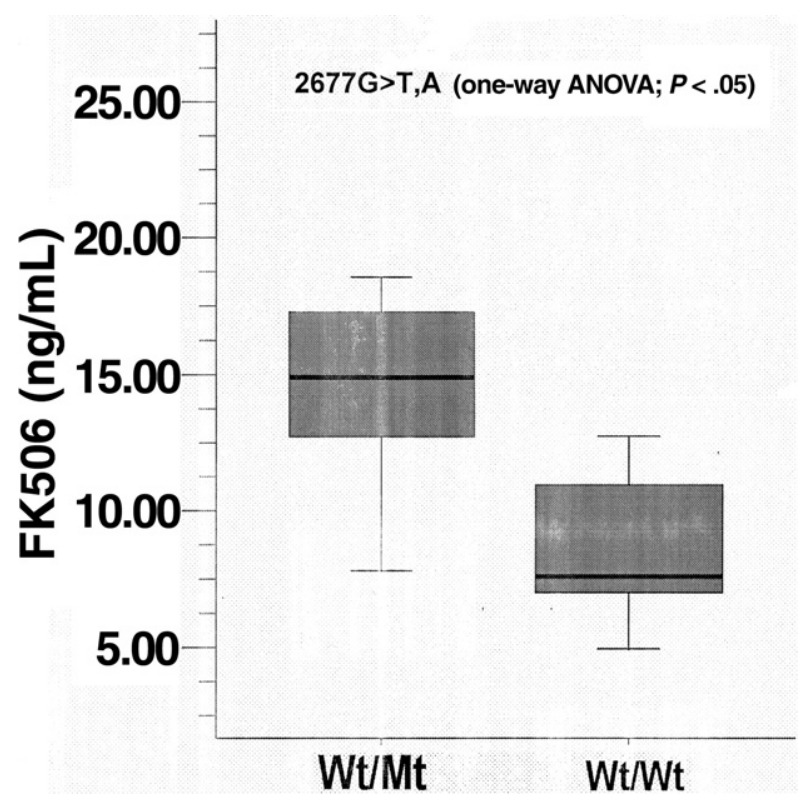

Fig 2. Correlation between plasma levels of FK506 $(\mathrm{ng} / \mathrm{mL})$ and the SNP 2277G > T,A in exon 21 of the MDR1 gene. Concentration distribution in accordance with the allelic variation. Wt, wild-type genotype; Mt, mutant genotype. 


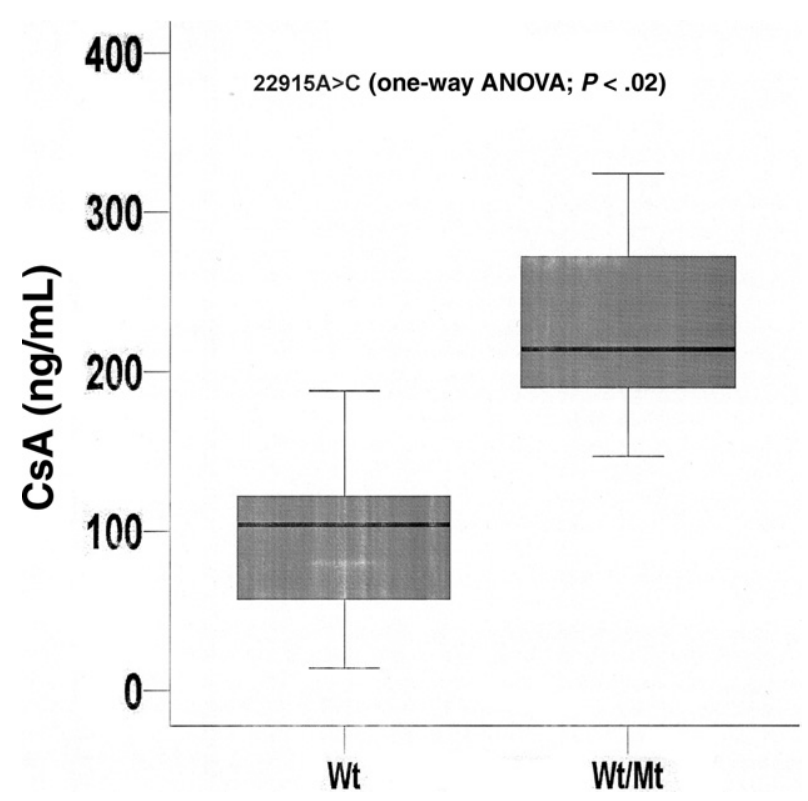

Fig 3. Correlation between the plasma levels of CsA (ng/mL) and the SNP 22915A>C in exon 3 of the CYP3A5 gene. Concentration distribution in accordance with the allelic variation. Wt, wild-type genotype; Mt, mutant genotype. alternative to pharmacokinetic studies conducted posttransplantation, in which several blood samples must be obtained at regular intervals to determine the ideal dose to reach the optimal concentrations.

\section{REFERENCES}

1. Masuda S, Inuia K: An up-date review on individualized dosage adjustment of calcineurin inhibitors in organ transplant patients. Pharmacol Ther 112:184, 2006

2. Hoffmeyer S, Burk O, von Richter $\mathrm{O}$, et al: Functional polymorphisms of the human multidrugresistance gene: multiple sequence variations and correlation of one allele with P-glycoprotein expression and activity in vivo. Proc Natl Acad Sci U S A 97: 3473,2000

3. Wei-lin W, Jin J, Shu-sen Z, et al: Tacrolimus dose requirement in relation to donor and recipient $A B C B 1$ and $C Y P 3 A 5$ gene polymorphisms in Chinese liver transplant patients. Liver Transpl 12:775, 2006

4. Kuehll $\mathrm{P}$, Zhang J, Lin Y, et al: Sequence diversity in $C Y P 3 A$ promoters and characterization of the genetic basis of polymorphic CYP3A5 expression. Nat Genet 27:383, 2001

5. Anglicheau D, Verstuyft C, Laurent-Puig P, et al: Association of the Multidrug Resistance-1 gene single nucleotide polymorphisms with the tacrolimus dose requirements in renal transplant recipients. J Am Soc Nephrol 14:189, 2003 